\title{
Multivariable Predictive Functional Control of an Autoclave
}

\author{
Aleksander Preglej1,* - Igor Steiner ${ }^{1}$ - Sašo Blažič ${ }^{2}$ \\ 1 INEA d.o.o., Slovenia \\ 2 University of Ljubljana, Faculty of Electrical Engineering, Slovenia
}

\begin{abstract}
This paper presents the predictive functional control of an autoclave, which is designed, tested and compared in uni- and multivariable manners. The control of the autoclave is based on our previously developed mathematical model for an autoclave, where we dealt with the heat-transfer and pressure-changing processes. First, we presented the principles of the predictive control algorithm, which are easy to understand. Next, the basic principles of predictive control were extended to a multivariable manner, so we presented the control law of the multivariable predictive algorithm. Furthermore, we depicted the suggested tuning rules for both control algorithms, which normally give satisfactory results, considering the trade-off between robustness and performance. We implemented both predictive algorithms in the linearized and simplified autoclave model, where we applied faster tuning rules due to the need for faster closed-loop responses. For comparison we also designed and applied a classical compensating PI controller. The results show superior performance of the multivariable predictive approach. All three algorithms rise similarly quickly, but then the PI and the simple predictive controller slowly approach the desired value due to slower tuning because of the very noisy manipulated variable. The interactions in the autoclave model are not very strong, therefore by the interactions influence rejection, both predictive algorithms show similar performances, while the PI approach performs much worse. The multivariable predictive approach also proves its superior performance by the interactions influence rejection when controlling the processes with stronger interactions. We can conclude that the autoclave should be controlled as one multivariable process using a multivariable predictive functional approach.
\end{abstract}

Keywords: predictive control, multivariable control, autoclave, temperature, pressure, interactions

\section{INTRODUCTION}

The control of the autoclave is designed on the basis of a mathematical model of the autoclave developed in [1], where the paper deals with the various types of heat transfer, basic heat-transfer equations, heattransfer coefficients, heat flow, forced convection, conduction, thermal resistance, specific theories about dimensionless numbers like the Nusselt, Reynolds and Prandtl numbers, etc. The other process treated mathematically is pressure changing. The focus is the process inside the autoclave, which can be more simply described as heating, cooling and changing the pressure. Most of the used data are real and obtained from the autoclave manufacturer, but where this was not possible, the method of the model's response fitting to the measured data was used. At the end of the paper we conclude that the designed model is usable for a variety of process control, due to the obtained very similar simulated and real process responses, considering some simplifications and using a curvefitting procedure.

The similar heat transfer problems like natural and enhanced convection were studied in [2] and even more specific problems like radiation, conduction, and also natural and forced convection mechanisms were studied in [3]. In [4] the authors focus on the issue of heat treatment, where heat transfers were calculated using methodology based on inverse heat transfer, and similarly the inverse problem was used in [5] to approximate heat conduction.

In a real process, temperature and pressure control are treated as two independent control loops. The temperature is controlled continuously with two predictive functional controllers (PFCs) and pulse-width modulation of the heating with electrical heaters and cooling with a water cooler and an analogue valve. The pressure is discretely controlled with pressure increasing through the onoff valve and pressure decreasing through two on-off valves of different sizes. The advantage of predictive control is that it is simple to understand and easy to tune [6]. Predictive control [7] and [8] is based on a forecast of the process output signal at each sampling instant, where the forecast can be made implicitly or explicitly, based on a controlled process model. In the next step the control is selected such that it brings the predicted process output signal back to the reference signal in a way that minimizes the control error in the area between certain time horizons. Predictive control normally provides good performance, so it is not surprising that it is increasingly used in the industry [9] to [14].

First, the predictive functional control algorithm was developed for linear systems and then the basic idea was extended to nonlinear systems [15] to [20]. An example of industrial use of fuzzy predictive control is proposed in [21] to [23], where new Takagi-Sugeno proportional-integral predictive fuzzy 
controllers (PI-P-FCs) to speed control a class of servo systems are presented.

Like most industrial plants, the autoclave also exhibits a multivariable nature, which means that it has at least two inputs, two outputs and more than one variable that have to be controlled. In many cases more than one input variable is coupled with the outputs. The interactions between the temperature and the pressure in the autoclave cannot be neglected, so they were taken into account. Multivariable process control is well known and it was extensively studied in [24] and [25]. The fundamental principles of predictive functional control [7] were applied and extended to the multivariable case in [27]. In comparison with classical multivariable approaches, the main advantage of multivariable predictive functional control (MPFC) is in its simple design and high-quality control performance.

In the paper the predictive functional control of an autoclave in uni- (PFC) and multivariable (MPFC) manners is presented. The performances of both predictive algorithms are compared to the classical compensating PI approach. The results show the advantage of using the MPFC controller for multivariable autoclave processes.

The paper is organized as follows: in Section 1 the details of the autoclave model are given. In Sections 2 and 3 the univariable and multivariable predictive functional control are introduced, and in Section 4 controller tuning rules for both predictive control algorithms are presented. The implementation of both predictive control algorithms and the compensating PI approach is depicted in Section 5 and the results are collected in Section 6. Finally, the conclusions are drawn in Section 7.

\section{DETAILS OF THE AUTOCLAVE MODEL}

An autoclave is a pressure vessel in a cylindrical form [1] where composite semi-products are heated at selected temperatures and under high pressure, and so under the applied conditions become harder and therefore of a higher quality. In the autoclave the working pressure is up to 7 bar and the working temperature is up to $180^{\circ} \mathrm{C}$.

The autoclave is made of stainless steel and isolated with mineral wool and an isolating aluminium coat. The volume of the autoclave is 5600 litres. The autoclave is heated with electrical heaters with a power up to $110 \mathrm{~kW}$ and cooled with an inner cooler with a power up to $73 \mathrm{~kW}$ using cooling water. The pressure in the autoclave is increased and decreased by a flow of compressed air. A centrifugal ventilating fan on the back of the autoclave with a water-cooled mechanical axle washer and an electromotor drive outside the autoclave provide the air circulation.

The mathematical model of the autoclave's heating is built using heat flows and energy-balance equations, where the heat-transfer coefficients, areas of thermal conductivity, resistances of thermal conductivity, material masses, specific heat capacities, thermal conductivities, convection coefficients, characteristic lengths and Nusselt numbers are defined. In addition to the influence of the conductance on the heat transfer, forced convection is also significant. The delay with the heating is $30 \mathrm{~s}$.

The cooling process is very similar to the heating process. The only difference is the source, which is heaters for heating and a cooler for cooling. All the other heat flows are the same. The mathematical model of the autoclave's cooling is again built using heat flows and energy-balance equations, where similar parameters and coefficients are defined. The delay with the cooling is also $30 \mathrm{~s}$.

The pressure in the autoclave is increased with compressed air through the entry on-off valve and decreased by letting the air out through two exit onoff valves of different sizes. The valves are modelled as analogue valves, where both exit valves are considered as a single valve with a larger dimension. The mathematical model of the pressure changing is built using mass flows and mass-balance equations. The delay with the pressure changing is $1 \mathrm{~s}$.

Some of the model parameters were first estimated and then optimized with the method of the model's response fitting to the measured data with the criterion function of the sum of squared errors, described with symbols as follows:

$$
\theta_{\text {p.set }}=\operatorname{argmin}\left(\sum\left(y_{\text {process }}-y_{\text {model }}\right)^{2}\right) \text {. }
$$

In Eq. (1) the following notations are included: $\theta_{p . s e t}$ is the set of parameters, $y_{\text {process }}$ the real process output and $y_{\text {model }}$ is the mathematical model output.

The set of parameters, which were optimized using mentioned method, consists of the coefficients in Nusselt numbers calculations $x$ and $q$, metal and coat masses $m_{m e}$ and $m_{c}$, metal and mineral wool thicknesses $l_{m e}$ and $l_{w}$, air circulation velocities $u$ and $v$, and surface between the air in the autoclave and the metal $S_{\text {ame }}$ by the autoclave heating model.

By the autoclave cooling model the set of optimized parameters consists of the volume flow of the cooling water $\Phi_{c w i}$, cooler surface $S_{c w a}$, heattransfer coefficient between the cooling water and the air in the autoclave $K_{c w a}$, and again metal thickness $l_{m e}$ 
and surface between the air in the autoclave and the metal $S_{\text {ame }}$.

The set of optimized parameters by the autoclave pressure changes model consists of nonlinearity $K_{n l}$ and the valve constants $K_{\text {in }}$ and $K_{\text {out }}$.

\section{PREDICTIVE FUNCTIONAL CONTROL}

Since they are natural and can be rapidly understood, the basic principles of PFC control are very solid and easy to understand [9]. The algorithm is based on the explicit use of a dynamic process model to predict the future process-output behaviour over a finite horizon and to evaluate control actions in order to minimize the chosen cost function.

For each time constant the optimal control sequence according to the criterion is obtained, but only the first element is used and applied.

The control goal is to determine the future control action so that the predicted output trajectory coincides with the reference trajectory, which is given in the form of the reference model. The single coincidence point (horizon) is assumed and is called the coincidence horizon $(H)$. The prediction is calculated using the known strategy of mean level control under the assumption of constant future manipulated variables $u(k)=u(k+1)=\ldots=u(k+H-1)$.

The main idea of the PFC is the equivalence of the objective increment of the process $\Delta_{p}$ and the model output increment $\Delta_{m}$, from which the control law of the PFC is obtained:

$$
u(k)=\frac{\left(1-a_{r}^{H}\right)(w(k)-y(k))}{\frac{b_{m}}{1-a_{m}}\left(1-a_{m}^{H}\right)}+\frac{y_{m}(k)}{\frac{b_{m}}{1-a_{m}}},
$$

where $a_{m}$ can be written as $a_{m}=e^{-T s / T m}$, in which $T_{m}$ is the model time constant and $T_{s}$ stands for the sampling time, and $a_{r}$ can be written as $a_{r}=e^{-T s / T r}$, in which $T_{r}$ is the reference model time constant. Furthermore, $b_{m}$ is the model gain, $w$ is the reference signal, $y$ is the process output and $y_{m}$ is the process model output.

If the process has a time delay, the control law is modified according to Smith's predictor principle [26]:

$$
u(k)=\frac{\left(1-a_{r}^{H}\right)\left(w(k)-y^{\prime}(k)\right)}{\frac{b_{m}}{1-a_{m}}\left(1-a_{m}^{H}\right)}+\frac{y_{m}(k)}{\frac{b_{m}}{1-a_{m}}},
$$

where $y^{\prime}(k)=y(k)+y_{m}(k)-y_{m d}(k)$, in which $y_{m d}$ is the delayed model output.

\section{MULTIVARIABLE PREDICTIVE FUNCTIONAL CONTROL}

The basic principles of predictive functional control were extended to the multivariable manner [27]. The problem of delays in the plant is circumvented by constructing an auxiliary variable that serves as the output of the plant if there are no delays present. A discrete undelayed process model must be given in the state-space form with the matrices $A_{m}, B_{m}$ and $C_{m}$ :

$$
\begin{gathered}
x_{m}(k+1)=A_{m} x_{m}(k)+B_{m} u(k), \\
y_{m}^{0}(k)=C_{m} x_{m}(k),
\end{gathered}
$$

where $x_{m}$ is the model state vector, $u$ is the model's input and $y_{m}^{0}$ is the undelayed model output.

The control goal is the same as in Section 2 and the reference trajectory is again given in the form of the reference model. The prediction is also calculated using the known strategy of mean level control under the assumption of constant future manipulated variables.

Next, we can obtain the $H$-step-ahead prediction of the undelayed model:

$$
y_{m}^{0}(k+H)=C_{m}\left[A_{m}^{H} \cdot x_{m}(k)+M_{A I B} \cdot u(k)\right],
$$

where $M_{A I B}=\left(A_{m}{ }^{H}-I\right)\left(A_{m}-I\right)^{-1} B_{m}$, in which $I$ is identity matrix.

The reference model is given as:

$$
\begin{gathered}
x_{r}(k+H)=A_{r} \cdot x_{r}(k)+B_{r} w(k), \\
y_{r}(k)=C_{r} \cdot x_{r}(k),
\end{gathered}
$$

where $x_{r}$ is the reference model state vector, $w$ is the reference signal and $y_{r}$ is the reference model output. The matrices $A_{r}, B_{r}$ and $C_{r}$ of the reference model must satisfy the equation:

$$
C_{r}\left(I-A_{r}\right)^{-1} B_{r}=I,
$$

which enables reference trajectory tracking due to a unity gain for each channel. If a first-order reference model is used its matrices become diagonal. Furthermore, we can choose $C_{r}=I$ and so it must be $B_{r}=I-A_{r}$. The reference-model prediction can then be given as:

$$
y_{r}(k+H)=A_{r}^{H} y_{r}(k)+\left(I-A_{r}^{H}\right) w(k),
$$

where a constant and bounded reference signal $w(k+i)=w(k), i=1, \ldots, H$ is assumed.

If the main idea of the MPFC is taken into account, the reference-trajectory tracking $y_{r}(k+i)=y_{p}{ }^{0}(k+i), i=1, \ldots, H$, next equalise the 
objective increment vector $\Delta_{p}$ and the model output increment vector $\Delta_{m}$ :

$$
y_{r}(k+H)-y_{p}^{0}(k)=y_{m}^{0}(k+H)-y_{m}^{0}(k),
$$

and do some further calculations, we obtain the control law of the MPFC controller:

$$
u(k)=G_{0}^{-1}\left(\left(I-A_{r}^{H}\right) e^{\prime \prime}(k)+M_{A C} x_{m}(k)\right),
$$

where $e^{\prime \prime}(k)=w(k)-y_{p}(k)+y_{m}(k), M_{A C}=$ $=A_{r}{ }^{H} C_{m}-C_{m} A_{m}{ }^{H}$ and $G_{0}=C_{m}\left(A_{m}{ }^{H}-I\right)\left(A_{m}-I\right)^{-1} B_{m}$.

The control law is realizable if $G_{0}$ is nonsingular, which is true when the plant is stable, controllable and observable. In other words, the MPFC control law can only be implemented for stable open-loop systems.

\section{CONTROLLER TUNING RULES}

Predictive functional controllers are quite easy to tune because there are just a few parameters to set. The first requirement is to have the plant model. With the PFC this means having a model time constant $T_{m}$, a model gain $K_{m}$ and a model delay $D_{m}$. On the other hand, with the MPFC this means having the model written in the undelayed discrete state-space form with the matrices $A_{m}, B_{m}$ and $C_{m}$.

From here on only two controller parameters need to be tuned. With the PFC these are the reference model time constant $T_{r}$ and the coincidence horizon $H$. With the MPFC these are the discrete reference model matrix $A_{r}$ and again the coincidence horizon $H$. Note that the coincidence horizon $H$ must always be an integer value.

We investigated the predictive functional controller's behaviour and performance using the different parameter settings on various simple and complex models.

With the PFC we took into account the results from [6] and tested their relevance. Using these settings the control performance was good, so we used the $T_{r}$ rule and the bit fastened $H$ rule, which we can combine in $T_{r}=T_{m} / 10$ and $H=\operatorname{round}\left(T_{r} /\left(2 T_{s}\right)\right)$.

In addition, with the MPFC we examined the findings from [27], where the stability of the control algorithm regarding the parameter $H$ was studied. If $H$ is less than the maximum relative degree $\rho$ of the model $(H<\rho)$, the matrix $G_{0}$ becomes singular and the control law is not applicable. When $H$ is equal to $\rho(H=\rho)$ the obtained closed-loop control is stable only if all the open-loop transmission zeros are inside the unit circle. And when $H$ tends to infinity $(H \rightarrow \infty)$ the system matrix of the closed-loop system $A_{c}$ goes to $A_{m}$, from which it can be concluded that a stable control law could always be obtained for open-loop stable systems, even if some open-loop transmission zeros are outside the unit circle when a suitable coincidence horizon is used.

The discrete reference model matrix $A_{r}$ can be, using Eq. (7) and further assumptions, easily presented as a diagonal matrix of the reference model time constants in a continuous time:

$$
A_{r}=\left[\begin{array}{cccc}
a_{r 1} & 0 & \ldots & 0 \\
0 & a_{r 2} & \ldots & 0 \\
\vdots & \vdots & \ddots & \vdots \\
0 & 0 & \ldots & a_{r m}
\end{array}\right],
$$

where $m$ stands for the number of model states, $a_{r m}$ is the state matrix $(A)$, which in this case is a constant, of the discretized with $T_{S}$ continuous time state-space model with $A^{\prime}=-1 / T_{r m}, B^{\prime}=1 / T_{r m}, C^{\prime}=1$ and $D^{\prime}=0$.

Following that we can give the tuning rules for MPFC parameters like $T_{r m}=T_{m m} / 2.5$ and $H \geq \rho$, where $T_{m m}$ is the continuous time constant of the $m^{\text {th }}$ reference transfer function.

$H \geq \rho$ is valid for stable open-loop systems with all open-loop transmission zeros inside the unit circle. If some open-loop transmission zeros are outside the unit circle, the setting $H>\rho$ must be used.

In general, these tuning rules are default settings, which normally give satisfactory results, considering the trade-off between the robustness and performance of the controlled system. If our requirements are different, we can also set it higher or lower. However, caution is needed as a lower $T_{r}$ or $T_{r m}$ means a more tightened and faster control loop, and so the closedloop can become unstable. A lower $H$ also means a more tightened and faster control loop, so a lot of noise can be propagated through the system. The lower limit for $H$ is one, because normally the models have a maximum relative degree equal to one. A higher $H$ propagates less noise through the system, but also slows down the control loop.

\section{IMPLEMENTATION}

For the implementation of the control algorithms we had to linearize and simplify the built multivariable mathematical model of an autoclave [1]. A sampling time $\left(T_{S}\right)$ of 1 second was used. Univariable predictive control is designed based on a first-order transfer function of a process. We used the following approximations: 


$$
\begin{gathered}
G_{\text {heat }}(s)=\frac{0.0485}{63034 s+1} e^{-30 s}, \\
G_{\text {press }}(s)=\frac{4.01 e^{5}}{288 s+1} e^{-1 s},
\end{gathered}
$$

where $G_{\text {heat }}$ is the first-order transfer function of the heating process and $G_{\text {press }}$ is the pressure-changing process transfer function.

From the transfer functions in Eq. (12) and (13) we can easily obtain the model parameters for the time constant, gain and delay, which are $T_{m h}=63034$, $K_{m h}=0.0485$ and $D_{m h}=30$ for the heating process, respectively, and $T_{m p}=288, K_{m p}=4.01 e^{5}$ and $D_{m p}=1$ for the pressure-changing process, respectively.

For the reference-model time constant the tuning rules suggest $T_{r}=T_{m} / 10$ and $H=T_{r} / 2$, but the specialty of the real time autoclave process and the customer demands required faster closed-loop responses. Real-time experiments showed that the best results are obtained with the controller parameters $T_{r h}=180$ and $H_{h}=90$ for the heating process. The realtime pressure changing is discretely controlled, but for the purpose of the simulation and the comparison with the MPFC control, we also designed the PFC with the controller parameters $T_{r p}=11.5$ and $H_{p}=6$.

Since the difference between the heating and cooling processes is in fact only the source (the heaters by heating and the cooler by cooling), we used the same model to which we applied different source values. In spite of the controller limits, this refers to the heating from 0 to $110 \mathrm{~kW}$ of the heaters power and for the cooling from 0 to $-73 \mathrm{~kW}$ of cooler power. With the pressure-changing process for the pressure increasing we used the controller limits from 0 to 100 of inlet valve opening and for the pressure decreasing from 0 to -100 of outlet valve opening. The same source values and controller limits were used by the MPFC algorithm.

Multivariable predictive control is designed based on an undelayed continuous two-inputs twooutputs state-space model with matrices $A_{m}=a_{i j} ; i, j=$ $1, \ldots, 5, B_{m}=b_{i j} ; i=1, \ldots, 5, j=1, \ldots, 2$, and $C_{m}=c_{i j} ; i$ $=1, \ldots, 2, j=1, \ldots, 5$, where $a_{11}=-2.914, a_{12}=0.024$, $a_{13}=2.887, a_{14}=4.25 e^{-5}, a_{15}=1 e^{-6}, a_{21}=1.57 e^{-4}, a_{22}$ $=-1.57 e^{-4}, a_{31}=1.89 e^{-2}, a_{33}=-1.89 e^{-2}, a_{41}=1.02 e^{-5}$, $a_{44}=-1.02 e^{-5}, a_{51}=0.05, a_{55}=-3.5 e^{-3}, b_{11}=2.5 \mathrm{e}^{-4}$, $b_{52}=1400, c_{11}=1, c_{25}=1$ and all others are zero. These matrices are then used in the discrete form.

From the previously mentioned state-space model we estimated the time constants $T_{m 1}=63034$ and $T_{m 2}=288$. For the $m^{\text {th }}$ reference model time constant the tuning rules suggest $T_{r m}=T_{m m} / 2.5$, but again faster closed-loop responses are required. The simulation experiments showed that the best results are obtained with the controller parameters $T_{r 1}=40$ and $T_{r 2}=6$, which can be transformed to the following discrete reference model matrix $A_{r}$ :

$$
A_{r}=\left[\begin{array}{cc}
0.9753 & 0 \\
0 & 0.8465
\end{array}\right] \text {. }
$$

For the second controller parameter $H$ the tuning rules suggest $H \geq \rho$, where $\rho$ is 1 by the autoclave model. However, as mentioned above, a lower $H$ means a tighter and faster control loop, so a lot of noise is propagated through the system. Again, simulation experiments showed that the best results are obtained with $H_{m p f c}=10$.

For the comparison we also designed the classical compensating PI controller with the transfer function $G_{R}=K_{R}\left(T_{R} s+1\right) /\left(T_{R} s\right)$, where $K_{R}$ is the compensator gain and $T_{R}$ is the compensator time constant.

We used a compensator which zero cancels the system pole $\left(T_{R}=T_{P}\right)$ and so we get the openloop transfer function $G_{O L}=\left(K_{P} K_{R}\right) /\left(T_{P} s\right)$, where $T_{P}$ is the system time constant and $K_{P}$ is the system gain. The closed-loop transfer function is then $G_{C L}=\left(\left(T_{P} / K_{P} / K_{R}\right) s+1\right)-1$, from which the compensator gain using the system gains from Eqs. (12) and (13) can be determined in following equation:

$$
K_{R}=\frac{1}{K_{P} \alpha},
$$

where $\alpha$ is a coefficient between 0 and 1 , which tells us by how much the compensated system is speeded up (a lower $\alpha$ means a faster system).

As was stated previously, the compensator time constant is equal to the system time constant, which is 63034 by the temperature control regarding Eq. (12) and by the pressure control regarding Eq. (13) is 288 . $\alpha$ by the temperature control equals 0.0045 , which gives a compensator gain $K_{R t}=4.582 \mathrm{e}^{3}$, and further, $\alpha$ by the pressure control equals 0.42 , which gives a compensator gain $K_{R p}=5.938 \mathrm{e}^{-6}$.

\section{RESULTS}

We applied the same amount of white noise (with different variance for the temperature and pressure signals) to all three algorithms simulations on the system outputs. The simulation experiment, which lasted 8000 seconds, was carried out in the following steps: 
- we increased the pressure from atmospheric pressure ( 1 bar) to 5 bar at time $50 \mathrm{~s}$,

- next, we increased the temperature from room temperature $\left(24{ }^{\circ} \mathrm{C}\right)$ to $150{ }^{\circ} \mathrm{C}$ at time $1500 \mathrm{~s}$,

- then, we decreased the pressure to 3 bar at time $3000 \mathrm{~s}$,

- next, we again increased the pressure to 5 bar at time $4000 \mathrm{~s}$,

- $\quad$ next, we decreased the temperature back to room temperature $\left(24^{\circ} \mathrm{C}\right)$ at time $5000 \mathrm{~s}$,

- and finally, we decreased the pressure back to atmospheric pressure ( 1 bar) at time $6000 \mathrm{~s}$.

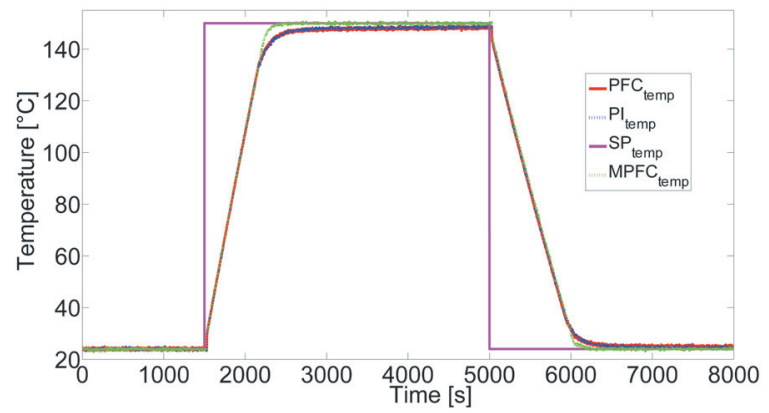

Fig. 1. $P I, P F C$ and MPFC performance comparison (PC); temperature set point in ${ }^{\circ} \mathrm{C}$ (magenta), PI (blue), PFC (red) and MPFC response (green line)

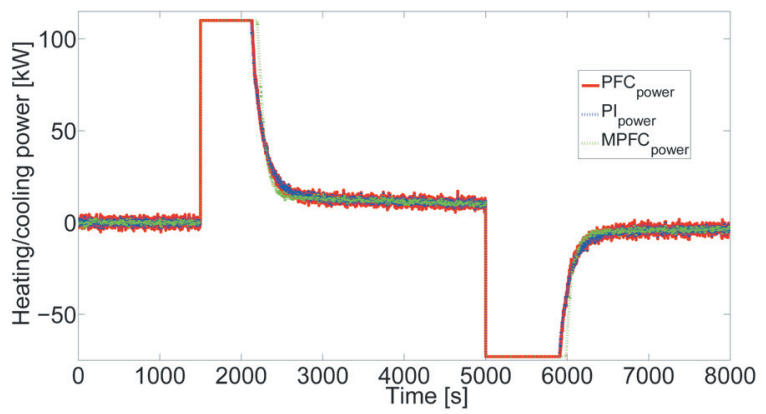

Fig. 2. PI, PFC and MPFC PC; PI (blue), PFC (red) and MPFC (green line) heating/cooling power in $\mathrm{kW}$

In Fig. 1 (temperature step responses) very similar responses of the PI and PFC algorithms can be observed, which rise relatively quickly but then very slowly approach the desired temperature. On the other hand, the MPFC algorithm is similarly quick, but it also reaches the desired value a lot more quickly than the other two algorithms. PI and PFC are tuned more slowly due to the very noisy manipulated variable during quicker tuning. In Fig. 2 the comparable noisy power manipulated variables of all three algorithms are presented. It is clear that the manipulated variable of the MPFC algorithm holds a little longer at the high/low limit, which explains reaching the desired temperature more quickly. The influence of the pressure change on the temperature here is minimal, so the disturbance cannot be seen in Figs. 1 and 2 .

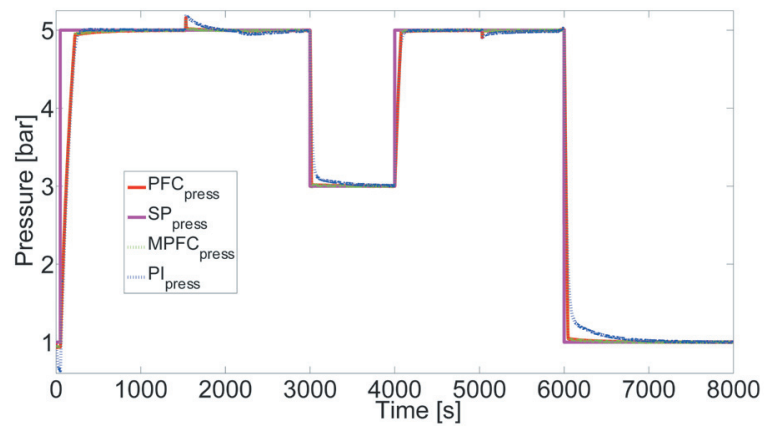

Fig. 3. PI, PFC and MPFC PC; pressure set point in bars (magenta), PI (blue), PFC (red) and MPFC response (green line)

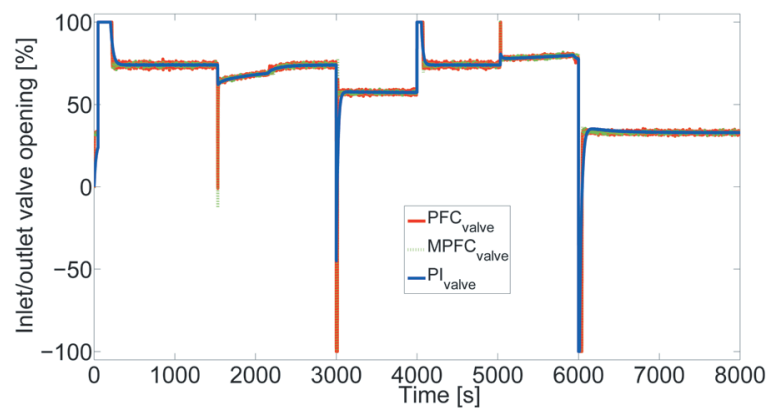

Fig. 4. $P I, P F C$ and MPFC PC; $P$ (blue), $P F C$ (red) and MPFC (green line) inlet/outlet valve opening in $\%$

In Fig. 3 (pressure step responses) we can see very similar responses of the PFC and MPFC algorithms, which quickly reach the desired pressure and also reject the influence of the temperature change on the pressure reasonably well, which can be seen around the times 1500 and 5000 s. On the other hand, the PI algorithm response is significantly slower, especially with the cooling, and also the rejection of the temperature change disturbance is much worse than with the other two algorithms. Again, in Fig. 4 the comparable noisy valve opening manipulated variables of all three algorithms are presented, where the PI manipulated variable is the least noisy, but also it does not reach the higher PFC and MPFC peaks for good temperature-change disturbance rejection.

\section{CONCLUSIONS}

For the needs of the classical compensating PI, univariable and multivariable predictive functional control system design we used a previously built mathematical model of an autoclave, which was linearized, then written in multivariable state-space 
model form and further simplified to two univariable first-order transfer-function models.

With the implementation of the PI, PFC and MPFC algorithms we followed the controller tuning rules and, where necessary, we used modified, faster rules.

The main advantage of the proposed MPFC algorithm is in the simple design, even in the case of delayed systems. In the results the MPFC approach proved its purpose because it showed the best performance. It allowed the fastest tuning, while the other two approaches performed with very noisy (not usable) manipulated variables at the same tuning speed.

As mentioned in the conclusions in [1], the interactions between the temperature and the pressure were taken into account. It can be said that also with the interactions disturbance rejection the MPFC showed superior performance, although the interactions in the autoclave model are not quite so strong. However, before implementing the MPFC approach to the autoclave model, we tested it on several simulated examples, where the interactions were much stronger. A direct comparison of the performance of the $\mathrm{PFC}$ and MPFC showed that the MPFC performs better with the interactions' disturbance rejection. In any case it can be concluded that the autoclave should be controlled as one multivariable process using a multivariable control approach, for example, MPFC.

As mentioned above [1], the autoclave model will also have to be additionally validated for the other real operating conditions and due to the very different regimes of operation some fuzzy-control approach, like the fuzzy-model-based multivariable predictive functional control (FMBMPC), should be implemented.

\section{ACKNOWLEDGEMENTS}

The operation part was financed by the European Union, European Social Fund. The operation implemented in the framework of the Operational Programme for Human Resources Development for the Period 2007 to 2013, Priority axis 1: Promoting entrepreneurship and adaptability, Main type of activity 1.1.: Experts and researchers for competitive enterprises.

\section{REFERENCES}

[1] Preglej, A., Karba, R., Steiner, I., Škrjanc, I. (2011). Mathematical model of an autoclave. Strojniški vestnik
- Journal of Mechanical Engineering, vol. 57, no. 6, p. 503-516, DOI:10.5545/sv-jme.2010.182.

[2] Venko, S., Vidrih, B., Pavlovič, E., Medved, S. (2012). Enhanced heat transfer on thermo active cooling wall. Strojniški vestnik - Journal of Mechanical Engineering, vol. 58, no. 11, p. 623-632, DOI:10.5545/ sv-jme.2012.436.

[3] Rek, Z., Rudolf, M., Zun, I. (2012). Application of CFD simulation in the development of a new generation heating oven. Strojniški vestnik - Journal of Mechanical Engineering, vol. 58, no. 2, p. 134-144, DOI:10.5545/sv-jme.2011.163.

[4] Taraba, B., Duehring, S., Španielka, J., Hajdu, Š. (2012). Effect of agitation work on heat transfer during cooling in oil ISORAPID 277HM. Strojniški vestnik Journal of Mechanical Engineering, vol. 58, no. 2, p. 102-106, DOI:10.5545/sv-jme.2011.064.

[5] Gostimirović, M., Sekulić, M., Kopač, J., Kovač, P. (2011). Optimal control of workpiece thermal state in creep-feed grinding using inverse heat conduction analysis. Strojniški vestnik - Journal of Mechanical Engineering, vol. 57, no. 10, p. 730-738, DOI:10.5545/ sv-jme.2010.075.

[6] Dovžan, D., Škrjanc, I. (2009). Self-tuning algorithms for predictive functional controller. Electrotechnical Review, vol. 74, no. 4, p. 205-210.

[7] Richalet, J. (1993). Industrial application of model based predictive control. Automatica, vol. 29, no. 5, p. 1251-1274, DOI:10.1016/0005-1098(93)90049-Y.

[8] Gerkšič, S., Strmčnik, S., Boom, T.V.D. (2008). Feedback action in predictive control: an experimental case study. Control Engineering Practice, vol. 16, no. 3, p. 321-332, DOI:10.1016/j.conengprac.2007.04.012.

[9] Dovžan, D., Škrjanc, I. (2012). Control of mineral wool thickness using predictive functional control. Robotics and Computer-Integrated Manufacturing, vol. 28, no. 3, p. 344-350, DOI:10.1016/j.rcim.2011.10.004.

[10] Qin, S.J., Badgwell, T.A. (2003). A survey of industrial model predictive control technology. Control Engineering Practice, vol. 11, no. 7, p. 733-764, DOI:10.1016/S0967-0661(02)00186-7.

[11] Vivas, A., Poignet, P. (2005). Predictive functional control of a parallel robot. Control Engineering Practice, vol. 13, no. 7, p. 863-874, DOI:10.1016/j.conengprac.2004.10.001.

[12] Karer, G., Škrjanc, I., Zupančič, B. (2008). Selfadaptive predictive functional control of the temperature in an exothermic batch reactor. Chemical Engineering and Processing, vol. 47, no. 12, p. 23792385, DOI:10.1016/j.cep.2008.01.015.

[13] Škrjanc, I. (2008). Self-adaptive supervisory predictive functional control of a hybrid semi-batch reactor with constraints. Chemical Engineering Journal, vol. 136, no. 2/3, p. 312-319, DOI:10.1016/j.cej.2007.04.012.

[14] Likar, B., Kocijan, J. (2007). Predictive control of a gas-liquid separation plant based on a Gaussian process model. Computers \& Chemical Engineering, 
vol. 31, no. 3, p. 142-152, DOI:10.1016/j. compchemeng.2006.05.011.

[15] Clarke, D. (1994). Advances in Model-Based Predictive Control. Oxford University Press, New York.

[16] Škrjanc, I., Matko, D. (2000). Predictive functional control based on fuzzy model for heat-exchanger pilot plant. IEEE Transactions on Fuzzy Systems, vol. 8, no. 6, p. 705-712, DOI:10.1109/91.890329.

[17] Blažič, S., Škrjanc, I. (2007). Design and stability analysis of fuzzy model-based predictive control - a case study. Journal of Intelligent and Robotic Systems, vol. 49, no. 3, p. 279-292, DOI:10.1007/s10846-0079147-8.

[18] Dovžan, D., Škrjanc, I. (2010). Predictive functional control based on an adaptive fuzzy model of a hybrid semi-batch reactor. Control Engineering Practice, vol. 18, no. 8, p. 979-989, DOI:10.1016/j. conengprac.2010.04.004.

[19] Causa, J., Karer, G., Núñez, A., Sáez, D., Škrjanc, I., Zupančič, B. (2008). Hybrid fuzzy predictive control based on genetic algorithms for the temperature control of a batch reactor. Computers \& Chemical Engineering, vol. 32, no. 12, p. 3254-3263, DOI:10.1016/j. compchemeng.2008.05.014.

[20] Škrjanc, I., Matko, D. (2001). Fuzzy predictive functional control in the state space domain. Journal of Intelligent and Robotic Systems, vol. 31, p. 283-297, DOI:10.1023/A:1012011010623.
[21] Precup, R.-E., Preitl, S., Faur, G. (2003). PI predictive fuzzy controllers for electrical drive speed control: methods and software for stable development. Computers in Industry, vol. 52, no. 3, p. 253-270, DOI:10.1016/S0166-3615(03)00130-1.

[22] Precup, R.-E., Preitl, S. (2004). Optimisation criteria in development of fuzzy controllers with dynamics. Engineering Applications of Artificial Intelligence, vol. 17, no. 6, p. 661-674, DOI:10.1016/j. engappai.2004.08.004.

[23] Precup, R.-E., Preitl, S., Korondi, P. (2007). Fuzzy controllers with maximum sensitivity for servo systems. IEEE Transactions on Industrial Electronics, vol. 54, no. 3, p. 1298-1310, DOI:10.1109/TIE.2007.893053.

[24] Rosenbrock, H.H. (1970). State-space and Multivariable Theory. Thomas Nelson and Sons Ltd, London.

[25] Maciejowski, J.M. (1989). Multivariable Feedback Design. Addison-Wesley, Workingham.

[26] Smith, O.J.M. (1957). Close control of loops with dead time. Chemical Engineering Progress, vol. 53, no. 1, p. 217-219.

[27] Škrjanc, I., Blažič, S., Oblak, S., Richalet, J. (2004). An approach to predictive control of multivariable time-delayed plant: Stability and design issues. ISA Transactions, vol. 43, no. 5, p. 585-595, DOI:10.1016/ S0019-0578(07)60170-0. 